\title{
The contribution of differential scanning calorimetry for the study of peptide/lipid in-
}

\section{teractions}

\author{
Marie-Lise Jobin ${ }^{\mathrm{a}}$, Isabel D. Alves ${ }^{\mathrm{b} *}$,
}

${ }^{a}$ Institute for Pharmacology and Toxicology, Rudolf Virchow Center - Bio-Imaging Center, University of Würzburg, Versbacher Str. 9, 97078 Würzburg, Germany

${ }^{\mathrm{b}}$ Chimie et Biologie des Membranes et Nanoobjets, CBMN CNRS UMR 5248, Université Bordeaux 1, Allée Geoffroy de Saint Hilaire, 33600 Pessac, France;

* Corresponding author: i.alves@cbmn.u-bordeaux.fr

\section{Summary}

Membrane active peptides include a variety of molecules such as antimicrobial (AMP), cell penetrating (CPP), viral and amyloid peptides that are implicated in several pathologies. They constitute important targets because they are either at the basis of novel therapies (drug delivery for CPPs or antimicrobial activity for AMPs) or they are the agents causing these pathologies (viral and amyloid peptides). They all share the common property of interacting with the cellular lipid membrane in their mode of action. Therefore a better understanding of the peptide/lipid (P/L) interaction is essential to help decipher their mechanism of action. Among the different biophysical methods that can be used to fully characterize P/L interactions, differential scanning calorimetry (DSC) allows determining the peptide effect on the lipid phase transitions, a property that reflects the $\mathrm{P} / \mathrm{L}$ interaction mode. A general protocol for classical DSC experiments for P/L studies will be provided.

\section{Keywords}

Membrane active peptides, peptide/lipid interaction, differential scanning calorimetry, lipid phase transition, thermodynamic behavior

Running head: DSC studies of P/L interactions 


\section{Introduction}

Membrane lipid properties (fluidity, thickness, ordering, etc) vary with temperature. The lipid membrane undergoes transition states as the temperature increases and the temperatures at which those occur are called phase transition temperatures. Lipid phase transitions are totally reversible. As the hydrocarbon length is increased, Van der Waals interactions become stronger requiring more energy to disrupt the ordered packing and arising to higher transition phase temperatures. When the number of lipid acyl chain unsaturation increases, the transition temperature decreases due to a disruption of acyl chain packing.

For lipids forming lamellar phases, at low temperatures lipids are in the gel phase (L $\left.\beta^{\prime}\right)$, a rigid phase where lipids are highly ordered and closely packed. As the temperature increases the tilting of lipid headgroups changes and lipids enter the rippled phase $\left(\mathrm{P} \beta \beta^{\prime}\right)$. In this intermediate phase, the bilayer is still highly ordered with low degree of fluidity and has a series of ripples along the surface. The phase transition associated is called the pre-transition and the temperature at which it occurs the pre-transition temperature $\left(\mathrm{T}_{\text {pre }}\right)$ (Figure 1). The pretransition is often only visible for single component lipid vesicles and tends to be absent when a mixture of lipids is studied. At higher temperatures, lipids undergo the main phase transition to a liquid disordered phase (L $\alpha$ ) that is accompanied by an increase in lipid disordering, decrease in bilayer thickness and lateral expansion [1]. The main transition is due to a cooperative melting of the hydrocarbon changes. It includes a conformational change of the hydrocarbon chains from all trans in the rigid gel state to a disordered state that allows gauche conformations. Accompanying these changes are a lateral expansion due to increased lipid mobility and a decrease in bilayer thickness. The main transition occurs at a higher temperature, is faster and associated with a larger enthalpy change than the pre-transition. 
Lipid phase transition properties are modulated by lipid physico-chemical properties as fatty acid chain length, degree of unsaturation, nature and size of their polar headgroup and by the buffer conditions (ionic strength, type of ions, $\mathrm{pH}$ ) and external factors as pressure $[2,3,4]$.

The lamellar transitions are the most studied since they are the most common in biological systems as they are adopted, among others, by phosphatidylcholines (PC), the most abundant phospholipid in eukaryotic cell membranes. An exception being for lipids that form hexagonal phases such as the phosphatidylethanolamines which, besides the lamellar phase possess an additional phase transition between lamellar $(\mathrm{L} \alpha)$ to hexagonal phase $\left(\mathrm{H}_{\mathrm{II}}\right.$; also called inverted micelle). The temperature phase transition between these two phases is called $\mathrm{T}_{\mathrm{H}}$, and occurs at higher temperatures than $\operatorname{Tm}[2,5,6,7]$.

One of the biophysical approaches that allow a full characterization of lipid phase transitions is Differential Scanning Calorimetry (DSC). DSC can measure phase transition temperatures $\left(T_{\text {pre }}, T_{m}, T_{H}\right)$ as well as enthalpy $(\Delta H)$ and cooperativity of the phase transitions by measuring the difference of temperature needed to compensate between the thermodynamic behaviors of two cells: one "inert" reference cell filled with aqueous buffer and one sample cell filled with the sample to be analyzed $[5,8,9]$. In a DSC measurement the difference in the amount of heat required to increase the temperature of a sample and reference are measured as a function of temperature. The basic principle underlying this technique is that, when the sample undergoes a physical transformation such as phase transitions, more or less heat will need to flow to it than the reference to maintain both at the same temperature. Whether less or more heat must flow to the sample depends on whether the process is exothermic or endothermic. By observing the difference in heat flow between the sample and reference, differential scanning calorimeters are able to measure the amount of heat absorbed or released during such transitions. This heat is then converted in specific heat capacity (Cp that is expressed in calories or joules per mol per ${ }^{\circ} \mathrm{C}$ ) that is usually employed in data presentation. 
Three parameters of interest can be extracted from DSC thermograms: the area under the transition peak, which is proportional to the enthalpy of the transition $(\Delta \mathrm{H})$ and directly correlates to the strength of van der Walls forces between the lipid fatty acid chains, the full width at half maximum (FWHM) of the transition peak $\left(\Delta \mathrm{T}_{1 / 2}\right)$ that reflects the cooperativity of the transition related with the number of molecules that undergo a transition simultaneously and the transition temperature itself $\left(\mathrm{T}_{\mathrm{pre}}, \mathrm{T}_{\mathrm{m}}, \mathrm{T}_{\mathrm{H}}\right)$.

DSC can be used to monitor phase transitions of single lipids, binary or simple lipid mixtures allowing mimicking more physiological membrane systems. One should keep in mind that for lipid samples composed of more than one lipid component, the lipids must be appropriately chosen to ensure proper miscibility (for reports on lipid miscibility see $[10,11,12,13,14])$. Additionally certain lipids such as cholesterol have a strong impact in the phase transition due to their natural intercalation between the fatty acid chains. Cholesterol broadens the endotherm and can even completely abolish the transition $[15,16]$, therefore their content must be kept low. At concentrations below 10\%, cholesterol induces minimal phase separation. Lipid miscibility will be further discussed below.

The mode of interaction of peptides with lipids can be accurately studied using DSC since this method is non-intrusive and doesn't implicate the use of any probe. As peptides interact with lipids they can induce changes in lipid properties as lipid packing, membrane fluidity, cooperativity of the transition and others that are reflected in the different parameters measured by the DSC experiment. Very generally, the pre-transition is very sensitive to all molecules that establish contacts with the lipid headgroups and tends to disappear when peptides are added. On the other hand, molecules that affect the main phase transition act at a deeper level than those that only affect the pre-transition, so with insertion below the headgroup level $[5,17]$. 


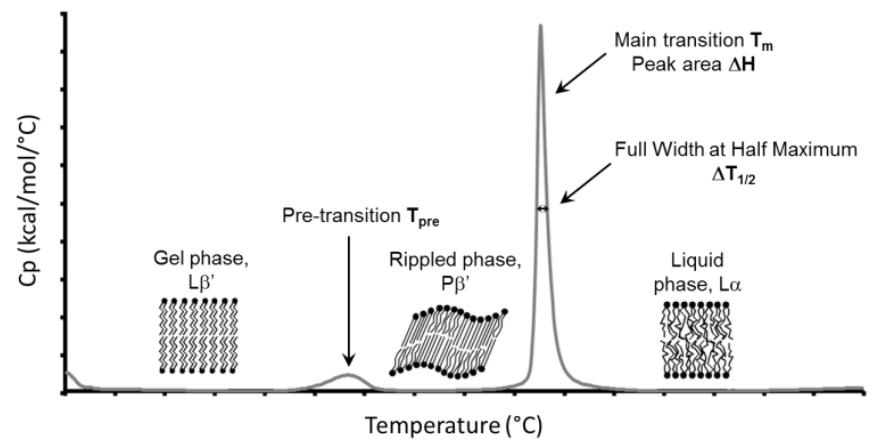

Fig. 1. Thermogram of a lamellar phase transition showing the three typical lipid phases (gel phase $L \beta^{\prime}$; Rippled phase $P \beta^{\prime}$ and liquid phase $L \alpha$ ) and the corresponding temperature transitions $\left(\mathrm{T}_{\text {pre }}\right.$ and $\left.\mathrm{T}_{\mathrm{m}}\right)$.

\section{Materials}

Lipids for the studies presented here were obtained from Avanti Polar Lipids (Alabama, USA).

The buffer used for DSC studies from our laboratory was $10 \mathrm{mM}$ Tris-HCl, $0.1 \mathrm{M}$ $\mathrm{NaCl}, 2 \mathrm{mM}$ EDTA, pH 7.6. Other buffers can be used considering that they do not undergo heat changes for the temperature ranges used in the measurements (see Note 1).

Peptides used in the studies were purchased from companies or synthesized by classical Solid-phase peptide chemistry procedures using Fmoc strategy, purified to $\geq 90 \%$ purity and lyophilized to a powder.

All DSC scans were obtained with a Nano DSC (TA Instruments) equipped with Ushape platinum cells. Other DSC instruments can be used.

\section{Methods}

\subsection{The lipid model system}


The most commonly used lipid model system used for DSC studies are multilamellar vesicles (MLVs) because these systems are very easy to prepare and are the most stable lipid model systems [18]. Moreover MLVs also result in a more homogeneous sample and in a higher and better resolved DSC signal than other types of liposomes as small and large unilamellar vesicles [5,19]. Regarding the composition of the lipid vesicles, one needs to choose lipids that are the most representatives of the in vivo system that one wants to mimic, but need to keep in mind the limitations associated with the method. Some additional considerations about the type and composition of the lipid model system are provided in Note 2 and 3.

1. A lipid film is prepared by dissolving the chosen lipid (1 $\mathrm{mg}$ when a single lipid is used and to investigate gel to fluid phase transition; for other conditions see Note 3) in minimal amount of chloroform and methanol (only if not soluble in chloroform alone, as it occurs often with anionic lipids) necessary to allow complete dissolution. For lipid mixtures, it is very important that lipids are thoroughly mixed and dissolved. To this aim the proportion of chloroform and methanol can be adjusted depending on the lipid to be dissolved. For example, if cholesterol is present the methanol content should be reduced to avoid crystallization of cholesterol. The same is valid for mixtures containing phosphatidylethanolamine. The film is evaporated under a flow of Nitrogen and then subjected to total evaporation under high vacuum to remove all traces of organic solvent ( $\geq 2 \mathrm{~h}$, depending on the system power).

2. The lipid film is hydrated by adding $1 \mathrm{ml}$ of the chosen buffer system (see Note 1) and then MLVs are spontaneously formed by strong vortex. For lipids with a lipid phase transition temperature $(\mathrm{Tm})$ that is considerably above room temperature, the sample should be warmed to a temperature above Tm to allow complete hydration and proper formation of MLVs. 
3. If unilamellar lipid vesicles (such as large ones, LUVs) are used, then after step 2, the MLVs are subjected to six steps of totally freezing/thawing (by immersing samples in liquid nitrogen followed by sample heating in a water bath at temperatures above the lipid phase transition). After that, the liposomes size is homogenized by passing the solution through a mini extruder equipped with $100 \mathrm{~nm}$ pore filter (Whatman). Due to a slight loss of lipid during the extrusion process $(\sim 5 \%)$, the lipid concentration should be determined by the Rouser method that can accurately quantify phosphate content in samples [20].

\subsection{The DSC measurement}

The DSC instrument is composed of two cells, a reference and a sample cell. In the reference cell the exact same buffer used for the sample (blank) must be loaded. In the sample cell, the lipid solution or the lipid and peptide mixture is loaded. The volume of the cells varies from one instrument to another (the studies reported here were performed with a DSC with $300 \mu \mathrm{L}$ cells).

1. Blank measurement should be performed first. For that the same buffer should be loaded in both the reference and the cell sample. Before loading, the buffer solution should be degassed for about 15 minutes to remove possible air bubbles. The same applies for all samples to be loaded in the DSC cells. Air bubbles displace liquid and therefore reduce the heat capacity (yielding erroneous results). As air bubbles can dissolve into solution over time, this will result in aberrant increase in heat capacity each subsequent cycle as the bubble dissolves. After loading the DSC cell, the fact that the cell is kept under pressure ( $35 \mathrm{psi})$ minimizes air bubbles. The temperature scan measurement should be performed over a large temperature range (to include the temperature range that is used for samples to be measured) and at a rate of $1^{\circ} \mathrm{C} / \mathrm{min}$ with a delay of $10-15$ min between sequential scans in a series that allows for thermal equilibration. The cell has a U-shape, and to load the solution, one should use two 1 
$\mathrm{mL}$ pipettes whose tips have been modified to have a $1 \mathrm{~cm}$ soft Teflon ending that whose radius perfectly fits to the DSC cell sample entrance to allow good sealing. Even if the cell sample volume is of only $300 \mu \mathrm{L}$, it is ideal to use a volume of at least $500 \mu \mathrm{L}$ for the loading procedure as this will reduce the possibility of introducing air bubbles that can be deleterious for the measurements. After loading a cap is placed in one of the two openings of the cell, the other opening is left open. A minimum of two cycles of heating/cooling should be performed, more if they differ (no stability reached). A flat line (no heat exchange) should be observed. If this is not the case, this could arise from several factors including the cell that is not properly cleaned, the existence of buffer heat variations with temperature or other.

2. After blank measurement, the buffer loaded in the sample cell is removed with a pipette and the lipid sample is introduced in the cell by the same method described above (no need to change the solution in the reference cell). The same scan parameters used for the blank should be applied here. Typically, a scan rate of $1^{\circ} \mathrm{C} / \mathrm{min}$ is used at the temperature range to be investigated with several (3-4) alternating heating and cooling scans to allow the system to reach equilibrium (more scans can be added if needed). Because the data in the first and last $5^{\circ} \mathrm{C}$ of the scan range is often non exploitable due to baseline problems, one should keep that in mind and broader the temperature scan to take that into account. There is no need to unload and load the reference cell since the buffer solution used in this measurement is the same as in the blank. Indeed the reference cell only needs to be filled once at the beginning of a series of measurements. If several sets of experiments are being run with the same buffer in the reference cell, one needs to insure that no evaporation had occurred from that cell. Even if each cell is capped in one of the two openings, the other remains uncapped so evaporation is possible after long time periods.

3. Then to investigate the effect of the peptide on the lipid phase transition, the sample containing both the lipid and the peptide (at the appropriated P/L ratio, see Note 4) is loaded 
in the sample cell and the same parameters (temperature scan range and number of heating and cooling scans) should be applied. It is important to test whether the peptide alone undergoes thermal reactions for the temperature range of the measurement. This is usually not the case for small peptides (contrarily to large proteins), as their unfolding does not involve high enough thermal changes to be observed by DSC at the low concentrations used for these experiments (below $\mathrm{mM}$ range).

4. After performing a complete set of experiments (blank, lipid and lipid with peptide sample), the sample cell needs to be cleaned. The cleaning solution is flowed through the cell by connecting tubing that establishes a circuit between the cells entrance and the washing solution through a vacuum system. This ensures a continuous and fast flow of solution through the cells. A solution of detergent in water, typically $200 \mathrm{~mL}$ of $1 \%$ Hellmanex is passed first followed by thorough washing with $1 \mathrm{~L}$ of milliQ water. This cleaning procedure is sufficient for typical lipid and peptide samples. If this cleaning procedure is found to be insufficient, harsher cleaning conditions such as $4 \mathrm{M}$ sodium hydroxide followed by $50 \%$ formic acid can be applied.

5. Ideally the cells should never be left empty to minimize hydration problems that occur when cells are filled after being left dry for long periods of time. Therefore, one can load either water or the reference buffer in the cell. The caps can be cleaned with detergent solution and plenty of water. Be careful about organic solvents as some caps material are not compatible with those resulting in changes in the shape and size of the caps.

\subsubsection{Troubleshooting}

This is not an exhaustive list but rather a summary of most common problems that can be encountered during measurements and how to solve them. Some of them have been briefly mentioned along the protocol. 
- Buffer/buffer scans are inconsistent. Heat capacity increases in subsequent scans. This can be due to the presence of air bubbles in the solution. The solution should be degassed and reloaded again.

- There is an effective Y-offset in the heat capacity data. This can be caused by pressure variation during data acquisition. This can result from opening/reclosing the cell to introduce the sample. The pressure is never exactly the same after replacing the pressure cap. This can also result from caps that are getting overused and that do not tightly close anymore.

- Sample scans are not similar between them (when comparing among heating or among cooling). This can just be due to the fact that the sample is not yet equilibrated and that temperature scans affect sample properties and homogeneity. The solution is to continue heating/cooling scans until the thermograms are comparable, meaning that the solution reached thermal equilibrium.

- The pressure does not stabilize. This can be due to several problems: buffer volume in the cells is too low, the cap used to close of the apertures is old and does not cap correctly.

\subsection{Data analysis and interpretation: examples}

Data analysis for data obtained with a DSC TA Instrument is performed with the fitting program CPCALC provided by CSC. Other instruments usually provide their own software with instrument.

1. The baseline (blank) should be subtracted from the sample data. To avoid the introduction of different data treatment from the user between different data sets, it is advisable to use a flat baseline treatment and to treat the data within similar temperature scans. It is best to start with the lipid alone for which thermodynamic phase transition data has been well reported in the literature, so that data can be compared. After data subtraction with baseline, the following parameters are obtained regarding the different lipid phase transitions (pre- and 
main phase temperature transition for lamellar lipids): phase transition temperature, enthalpy and entropy associated with the transition.

In the case where a mixture of lipids is used or a peptide is added to the liposomes, the DSC signal can be broadened or even splitted in a "two-peak" transition which could correspond to a domain formation (lipid domain or poor/rich-peptide domain). A data analysis software can be used to perform the deconvolution of the thermogram and identify the transition parameters of the different domains.

2. The exact same procedure is performed for the thermograms corresponding to the lipid in presence of peptide.

\subsubsection{Information obtained from DSC analysis}

From the parameters obtained after data analysis presented above, information about the mode of interaction of the peptides with the lipid membrane is obtained. Here a short summary is provided about the different parameters and their meaning:

- Modification of the phase transition temperature. This indicates that the peptide changes the lipid physico-chemical properties that are responsible for such transition. For example if the peptide decreases the Tm of a gel to fluid phase transition, it means that the peptide favors the transition thus has a fluidizing effect on the membrane.

- Changes in the area of the transition peak. Since the area of the transition peak is directly correlated with the enthalpy of the transition, a decrease in the area indicates that the peptide perturbs the phase transition and decreases the enthalpy. For a gel to fluid phase transition this indicates that the peptide perturbs the fatty acid chain packing and decreases the van der Waals interaction due to intercalation between the fatty acid chains. In certain cases the phase transition peak is completely abolished which is very common for the pre-transition that is sensitive to molecular interactions. 
- Changes in the transition peak FWHM. This parameter is a measure of the cooperativity of the transition, the sharper the transition is, the more cooperative the transition. This parameter is directly correlated with the changes in the peak area and again an increase of this parameter reflects peptide insertion in between the fatty acid chains.

- Changes in the shape of the transition peak. Besides reflecting changes in the cooperativity of the transition, this parameter is related with homogeneity. The de-doubling of a single transition peak and/or appearance of new transition peaks is indicative of species heterogeneity. If the peptide does not distribute homogeneously among all lipids the appearance of two phase transition peaks corresponding to poor and rich-peptide domains occurs. Usually the sharper transition peak is associated with the peptide-poor lipid domains and the broader transition is attributed to peptide-rich lipid regions.

Alternatively, the appearance of new transition peaks can be due to peptide-induced appearance of new lipid supramolecular structures (liposomes of different size, micelles, etc).

- Relation between main transition $\Delta H$ changes upon increasing concentration of peptide. By plotting this data and extrapolating the line, at $\Delta \mathrm{H}=0$ one can obtain the number of lipid molecules removed from the cooperative chain melting transition by each molecule of peptide [8].

For more in deep information the reader is directed to extensive review articles on the subject $[8,9,21]$.

\subsection{Unusual applications/cases}

\subsubsection{Study of lipid mixtures}

While most commonly single lipid model systems are investigated by DSC, the method can be applied to the study of lipid mixtures. 


\subsubsection{Following lipid domain formation and peptide partition into different domains}

In the context of binary lipid mixtures, DSC has been used to follow domain formation in lipid membranes. It is well know that cellular lipid membranes are not laterally homogeneous and that domains exist that are either morphological different structures or that only differ in their physico-chemical properties such as membrane ordering properties and fluidity, as it is the case for lipid-ordered and disordered domains. DSC has been used to study domain formation, mainly through the construction of phase diagrams and their interpretation in terms of phase miscibility. In view of phase miscibility, DSC can be useful to follow lipids mixtures that are miscible or not. In the first case, miscible lipid mixtures can result in single lipid phase transitions (that can become broader) when lipids have Tm values that are close. For example, when mixing lipids with different fatty acid chain length, lipids with chain lengths that differ by less than four carbons are not miscible $[22,23]$ and result in very well separated phase transitions. Both miscible and non miscible lipid mixtures can be interesting tools to study the mechanism of action of membrane active peptides: 1) In the case of miscible lipid mixtures, DSC can be used to follow the peptide effect in lipid reorganization, namely the preferential recruitment of one of the lipids in the mixture; 2) For non miscible lipids that result in well separated phase transitions observed by DSC, the preferential interaction of a peptide with one of the two lipids can be determined by the changes induced in each transition after peptide addition. Moreover some peptides have the capacity to improve lipid miscibility (for details see [24,25,26,27]).

\section{Notes}

1. Ideal buffers for DSC measurements should undergo minimal heat capacity variation with temperature. Most commonly used buffers in the laboratory are acceptable as Tris, Glycine, phosphate, etc... Buffers whose pKa varies considerably with temperature such as Hepes can 
be problematic. One point that absolutely needs to be considered is that the buffer composition must be absolutely the same in the reference and in the cell as very small variations in ion content can greatly affect the heat capacity and data measurements.

2. The most commonly used lipid model systems for DSC measurements are MLVs, because it is a simple system that can easily prepared in all laboratories without need for special equipment. Additionally MLVs are very stable in time and the phase transitions produced are very sharp and due to the high cooperativity of the transition. Another possibility is to use large unilamellar liposomes (LUVs). These systems are less stable than MLVs but still stable long enough for the time required for the measurements to be acquired. One advantage in using LUVs is related with the fact that the P/L ratio can be properly controlled because in a LUVs the ratio of lipid present in the outer versus inner leaflet is known. For MLVs, since their size and amount of bilayers per object is heterogeneous, the P/L ratio cannot be properly determined. The only exception to that is if the peptides investigated are able to cross all lipid layers and distribute homogeneously between the different membranes. Alternatively, it is also common to add peptides to lipids before formation of liposomes, the peptide is added at the moment of preparing the lipid film, and therefore the peptide becomes evenly distributed among the different lipid layers. This can be advantageous if the peptide is not easily dissolved in polar solvents. In our laboratory we have privileged the addition of peptide to lipid after liposome formation because this procedure resembles best the biological system.

3. Regarding the composition of the lipid model system, one needs to choose a lipid whose phase transition temperature is above $0^{\circ} \mathrm{C}$ (the instrument cannot record below that unless a special solvent is used that does not freeze at this temperature). For lipid model systems composed of a single type of lipid and for the investigation of gel/fluid phase transition, $1 \mathrm{mg}$ of lipid is enough for a set of experiments. If lipid mixtures are used, the amount of lipid needed for DSC measurements may have to be adapted depending on the lipid phase transition and 
whether the lipids are miscible or not. To keep in mind that in DSC one cannot use very complex lipid mixtures because this will result in very broad and shallow phase transitions. For lipids that are totally non-miscible, in principle their phase transition properties are kept and thus there is no need to increase the amount of lipid used. If lipids are miscible, the resulting phase transition is going to become broader and thus less well resolved, therefore lipid concentrations must be increased so that the transition can be properly followed. Lipids such as cholesterol when added to phospholipids have the tendency to greatly broaden the transition, so again higher lipid concentrations are necessary. Similarly, if one is to investigate other lipid phase transitions than the gel/fluid, for example the transition of lamellar $\left(\mathrm{L}_{\alpha}\right)$ to hexagonal $\left(\mathrm{H}_{\mathrm{II}}\right)$ phase transition, because smaller energy changes are implicated in such phase transitions (smaller transition peak) then the amount of lipid must be increased. In the case of the $\mathrm{L}_{\alpha}$ to $\mathrm{H}_{\text {II }}$ phase transition of DiPOPE (dipalmitoleoyl phosphatidylethanolamine), $10 \mathrm{mg}$ of the lipid were used in reported studies from our laboratory for a reasonably quantifiable transition to be monitored [28].

4. DSC studies on $\mathrm{P} / \mathrm{L}$ interactions have used $\mathrm{P} / \mathrm{L}$ ratios varying from $1 / 500$ to $1 / 10$. The $\mathrm{P} / \mathrm{L}$ ratio at which an effect will be measurable by DSC depends on the nature and extent of the peptide-induced lipid perturbation which is related with the class of membrane active peptide used. In general when the appropriated lipid is chosen, considerable peptide effects can be observed at P/L ratios of 1/50. Care must be taken with the use of very high peptide concentrations because precipitation may occur perturbing DSC signal.

\section{References}

1. Cullis P.R. FDB, Hope M.J. (1996) Physical properties and functional roles of lipids in membranes. In: J.E. VDEaV, editor. Biochemistry of Lipids, Lipoproteins and Membranes: Elsevier. pp. 1-32.

2. Israelachvili JN, Mitchell DJ, Ninham BW (1977) Theory of self-assembly of lipid bilayers and vesicles. Biochim Biophys Acta 470: 185-201. 
3. Lee AG (1977) Lipid phase transitions and phase diagrams. I. Lipid phase transitions. Biochim Biophys Acta 472: 237-281.

4. Lee AG (1977) Lipid phase transitions and phase diagrams. II. Mictures involving lipids. Biochim Biophys Acta 472: 285-344.

5. McElhaney RN (1982) The use of differential scanning calorimetry and differential thermal analysis in studies of model and biological membranes. Chem Phys Lipids 30: 229259.

6. McIntosh TJ (1996) Hydration properties of lamellar and non-lamellar phases of phosphatidylcholine and phosphatidylethanolamine. Chem Phys Lipids 81: 117-131.

7. Epand RM, Bryszewska M (1988) Modulation of the bilayer to hexagonal phase transition and solvation of phosphatidylethanolamines in aqueous salt solutions. Biochemistry 27: 8776-8779.

8. McElhaney RN (1986) Differential scanning calorimetric studies of lipid-protein interactions in model membrane systems. Biochim Biophys Acta 864: 361-421.

9. Seelig J (2004) Thermodynamics of lipid-peptide interactions. Biochim Biophys Acta 1666: $40-50$.

10. H H (2004) The microcalorimetry of lipid membranes. J Phys Condes Matter 16: 441-467.

11. Jimenez-Monreal AM, Villalain J, Aranda FJ, Gomez-Fernandez JC (1998) The phase behavior of aqueous dispersions of unsaturated mixtures of diacylglycerols and phospholipids. Biochim Biophys Acta 1373: 209-219.

12. Epand RM, Bach D, Epand RF, Borochov N, Wachtel E (2001) A new high-temperature transition of crystalline cholesterol in mixtures with phosphatidylserine. Biophys $\mathrm{J} 81$ : 1511-1520.

13. Lewis RN, Zhang YP, McElhaney RN (2005) Calorimetric and spectroscopic studies of the phase behavior and organization of lipid bilayer model membranes composed of binary mixtures of dimyristoylphosphatidylcholine and dimyristoylphosphatidylglycerol. Biochim Biophys Acta 1668: 203-214.

14. Garidel P, Blume A (2000) Miscibility of phosphatidylethanolamine-phosphatidylglycerol mixtures as a function of $\mathrm{pH}$ and acyl chain length. Eur Biophys J 28: 629-638.

15. Raudino A (1995) Lateral inhomogeneous lipid membranes: theoretical aspects. Adv Colloid Interface Sci 57: 229-285.

16. Almeida PF (2009) Thermodynamics of lipid interactions in complex bilayers. Biochim Biophys Acta 1788: 72-85.

17. Riske KA, Barroso RP, Vequi-Suplicy CC, Germano R, Henriques VB, et al. (2009) Lipid bilayer pre-transition as the beginning of the melting process. Biochim Biophys Acta 1788: 954-963.

18. Lichtenberg D, Freire E, Schmidt CF, Barenholz Y, Felgner PL, et al. (1981) Effect of surface curvature on stability, thermodynamic behavior, and osmotic activity of dipalmitoylphosphatidylcholine single lamellar vesicles. Biochemistry 20: 3462-3467.

19. Mason JT, Huang C, Biltonen RL (1983) Effect of liposomal size on the calorimetric behavior of mixed-chain phosphatidylcholine bilayer dispersions. Biochemistry 22: 2013-2018.

20. Rouser G, Fkeischer S, Yamamoto A (1970) Two dimensional then layer chromatographic separation of polar lipids and determination of phospholipids by phosphorus analysis of spots. Lipids 5: 494-496.

21. Lohner K, Prenner EJ (1999) Differential scanning calorimetry and X-ray diffraction studies of the specificity of the interaction of antimicrobial peptides with membranemimetic systems. Biochim Biophys Acta 1462: 141-156. 
22. Leidy C, Wolkers WF, Jorgensen K, Mouritsen OG, Crowe JH (2001) Lateral organization and domain formation in a two-component lipid membrane system. Biophys J 80: 1819-1828.

23. Shimshick EJ, Kleemann W, Hubbell WL, McConnell HM (1973) Lateral phase separations in membranes. J Supramol Struct 1: 285-294.

24. Joanne P, Galanth C, Goasdoue N, Nicolas P, Sagan S, et al. (2009) Lipid reorganization induced by membrane-active peptides probed using differential scanning calorimetry. Biochim Biophys Acta 1788: 1772-1781.

25. Epand RM (2007) Detecting the presence of membrane domains using DSC. Biophys Chem 126: 197-200.

26. Epand RF, Wang G, Berno B, Epand RM (2009) Lipid segregation explains selective toxicity of a series of fragments derived from the human cathelicidin LL-37. Antimicrob Agents Chemother 53: 3705-3714.

27. Polozov IV, Polozova AI, Molotkovsky JG, Epand RM (1997) Amphipathic peptide affects the lateral domain organization of lipid bilayers. Biochim Biophys Acta 1328: 125-139.

28. Alves ID, Goasdoue N, Correia I, Aubry S, Galanth C, et al. (2008) Membrane interaction and perturbation mechanisms induced by two cationic cell penetrating peptides with distinct charge distribution. Biochim Biophys Acta 1780: 948-959. 